\title{
Frecuencia de patologías abdominales diagnosticadas mediante ecografía en la clínica Veterinaria del Sur Sabaneta, Antioquia
}

\section{Frequency of abdominal pathologies diagnosed by ultrasound at the Southern Veterinary Clinic, Sabaneta, Antioquia}

\author{
Jhonny Buitrago $\mathrm{M}^{1^{*}} \mathrm{MVZ}$; Juan Osorio $\mathrm{A}^{2} \mathrm{MV}$; Ana Cadavid $\mathrm{R}^{1}$ M.Sc. \\ ${ }^{1}$ Corporación Universitaria Remington, Facultad de Medicina Veterinaria, Programa de Medicina \\ Veterinaria, Grupo de Investigación GINVER, Medellín, Colombia. \\ ${ }^{2}$ Corporación Universitaria Remington, Facultad de Medicina Veterinaria, Especialización en \\ Imagenología de Pequeñas Especies, Medellín, Colombia.
}

\section{KEYWORDS:}

Abdominal ultrasound; gastric inflammation; hepatic ultrasound; small species.

\section{ABSTRACT}

Ultrasound in small animals has advanced enormously in recent years, often replacing conventional radiographic studies. Abdominal ultrasound is a diagnostic aid of great importance for veterinary clinics. At present, its implementation has increased because it is a non-invasive technique, fast and timely, which together with the clinical evaluation of the patient allows us to identify pathologies in a short period. The objective of this report is to identify the frequency of abdominal pathologies diagnosed by means of ultrasound, as well as the most affected organ. For this, information was collected from the database of ultrasound reports of the southern veterinary clinic, located in Sabaneta Antioquia, between December 2016 and April 2017, in which 119 abdominal ultrasounds were performed, of which $82 \%$ was developed in canines and $18 \%$ in felines. The gastroenteric tract being the most frequently affected $(23.33 \%)$, followed by the liver (16\%) and the spleen ( $8 \%)$. The most frequently reported pathology was gastric inflammation $(8 \%)$, followed by hepatitis $(7.33 \%)$ and gastroenteritis (4\%).

\section{RESUMEN}

La ecografía en pequeños animales ha avanzado enormemente en los últimos años, llegando a sustituir en muchos casos a los estudios radiográficos convencionales. La ecografía abdominal es una ayuda diagnóstica de gran importancia para las clínicas veterinarias, en la actualidad su implementación ha aumentado debido a que es una técnica no invasiva, rápida y oportuna, que junto a la evaluación clínica del paciente nos permite identificar patologías en un corto periodo. El objetivo de este reporte es identificar la frecuencia de las patologías abdominales diagnosticadas por medio de ultrasonido, así como el órgano más afectado. Para esto se tomó información de la base de datos de los reportes ecográficos de la Clínica Veterinaria del Sur, ubicada en Sabaneta Antioquia, entre diciembre de 2016 y abril de 2017, en las cuales se realizaron 119 ecografías abdominales, de ellas el $82 \%$ fue desarrollada en caninos y $18 \%$ en felinos, siendo el tracto gastroentérico el más frecuentemente afectado (23,33\%), seguido del hígado (16\%) y el bazo $(8 \%)$. La patología reportada con mayor frecuencia fue la inflamación gástrica $(8 \%)$, seguida de la hepatitis $(7,33 \%)$ y la gastroenteritis $(4 \%)$. 


\section{INTRODUCCIÓN}

La ecografía es una técnica de diagnóstico por imagen que permite valorar tamaño, forma y estructura de los tejidos blandos de manera no invasiva, por lo que se considera sencilla, rápida, económica e inocua tanto para el paciente como para el técnico, ya que no utiliza radiaciones ionizantes y no suele requerir la sedación del animal (DIEZ et al, 2004; MORENO et al., 2011; XIFRA y SERRANO, 2016), la exactitud en su interpretación depende de la diferenciación entre la anatomía normal y la anormal de los órganos evaluados, y al contrario de otras técnicas de imagen, es necesario realizar la interpretación en el momento del estudio (NYLAND et al., 2001), por lo que una buena técnica ecográfica requiere equipos de alta resolución, transductores de alta frecuencia y personal capacitado (DIEZ et al., 2004; XIFRA y SERRANO, 2016), ya que requiere un elevado nivel de destreza manual y de coordinación entre la mano y el ojo, así como un profundo conocimiento de la anatomía, fisiología y patofisiología de los diferentes efectos en distintos tipos de cuerpos, así como de la capacidad y limitaciones del equipo. Es importante para el ecografista adquirir un método minucioso y sistemático para el examen abdominal. Esto posibilita una identificación precisa de todos los órganos y sistemas, y un examen muy eficiente. (NYLAND et al., 2001).

En medicina humana la ecografía se ha tornado en una gran ayuda que ha permitido mejorar el desempeño clínico de los médicos, especialmente en el diagnóstico de patologías abdominales ya que permite observar y medir con precisión múltiples órganos difíciles de valorar con los métodos tradicionales (inspección, palpación, percusión y auscultación) (GARCÍA DE CASASOLA y TORRES, 2012). En la medicina de los animales de compañía es una herramienta fundamental para el diagnóstico de muchas patologías y el desarrollo de estudios especiales, por lo que existe una tendencia creciente en su uso, especialmente para el diagnóstico de patologías gastrointestinales, sustituyendo en parte el uso de otro tipo de técnicas imagenológicas como la radiografía contrastada. (DIEZ et al., 2004).

La ecografía abdominal es considerada como una modalidad útil en la exploración de un amplio rango de signos clínicos en péqueños animales. Varios estudios han descrito los hallazgos ultrasonográficos relacionados a condiciones específicas y se ha demostrado una buena correlacion entre los hallazgos ultrasonográficos de la cavidad abdominal y los obtenidos mediante laparotomía exploratoria, demostrando su utilidad en la detección anatómica de lesiones primarias lo que hace de esta técnica una herramienta útil en el diagnóstico prequirúrgico de alteraciones abdominales (PASTORE et al., 2007). A pesar de esto son pocos los artículos encontrados en la literatura científica que reporten la frecuencia de patologías diagnosticadas mediante ecografía en la práctica clínica, pues los estudios se centran en la efectividad de diagnóstico para órganos o patologías específicas, y no de la cavidad abdominal en su totalidad, ni en la casuística atendida en centros veterinarios por lo que este artículo busca reportar las principales alteraciones ecográficas diagnosticadas en una clínica veterinaria dedicada a la atención de mascotas resaltando así la importancia de esta ayuda diagnóstica en la práctica veterinaria.

\section{MATERIALES Y MÉTODOS}

Se realizó un estudio prospectivo de las ecografías abdominales realizadas en la Clínica Veterinaria del Sur entre diciembre de 2016 y abril 2017. La clínica está Ubicada en el municipio de Sabaneta Antioquia, a 14 $\mathrm{Km}$ al sur de Medellín en las coordenadas 609'03" de latitud Norte y 75'36'54" de longitud Oeste.

Las ecografías fueron realizadas con un ecógrafo Mindray $®$ Z5 Vet, con transductor micro-convexo con frecuencias de 5 a 8 $\mathrm{MHz}$, la técnica fue ejecutada por un médico veterinario especialista con entrenamiento en ecografía abdominal.

Para la ejecución de la técnica ecográfica se utilizó alcohol comercial como medio de conducción, la tricotomía sólo se realizó en los casos que fueron autorizados por los propietarios. El desarrollo de la técnica se ejecutó según lo descrito por Xifra (2015), para lo cual los pacientes se posicionaron en decúbito supino y se realizó la exploración de forma circular en el sentido antihorario iniciando en la zona hipogástrica, en donde se valoró inicialmente la vejiga urinaria y se realizaron los ajustes del equipo, se evalúa el cuerpo del útero en las hembras, la próstata en los machos y los linfonodo ilíacos, 
posteriormente se desplaza el trasductor hacia el mesogastrio izquierdo en donde se evalúa el colon descendente y se continúa hacia craneal para evaluar riñón, glándula adrenal izquierda, ovario izquierdo en las hembras y el bazo, desde allí se realiza un movimiento hacia medial ubicándose en el epigastrio, más específicamente en la región xifoidea, en donde se visualizó el hígado, la vesícula biliar y el estómago; para finalizar se continúa hacia el mesogastrio derecho para evaluar el riñón, glándula adrenal derechos, duodeno y el área pancreática; en machos fueron también evaluados los testículos (XIFRA y GARCÍA, 2015).

Para el análisis se tuvieron en cuenta como criterios de inclusión las ecografías abdominales de animales de compañía que contaran con una exploración abdominal completa y con reporte oficial del médico veterinario, no se consideraron ecografías realizadas en otras especies animales, ecografías de urgencia, ecografía de órganos específicos o de otras regiones anatómicas, también fueron excluidos todos los reportes en los cuales no se realizara inspección de todos los órganos abdominales.

Con la información obtenida se elaboró una base de datos en Microsoft Excel versión 2017 y se realizó a partir de estadística descriptiva de la muestra evaluada y las principales alteraciones diagnosticados por ecografía, así como de los principales órganos afectados.

\section{RESULTADOS}

Durante el periodo de estudio se realizaron 119 ecografías abdominales que cumplieron con los criterios de inclusión, de estas el $82 \%$ (97) se realizaron en caninos y el $18 \%$ (22) en felinos (Tabla 1).

Tabla 1. Distribución de las ecografías abdominales realizadas en la Clínica Veterinaria del Sur según la especie.

\begin{tabular}{ccc}
\hline Especie & $\begin{array}{c}\text { Frecuencia } \\
\text { absoluta }\end{array}$ & $\begin{array}{c}\text { Frecuencia } \\
\text { relativa }\end{array}$ \\
Caninos & 97 & $82 \%$ \\
Felinos & 22 & $18 \%$ \\
\hline Total & 119 & $100 \%$ \\
\hline
\end{tabular}

En las ecografías realizadas durante el periodo de estudio no se detectó ninguna alteración ecográfica en un $17 \%$ de los casos, el $83 \%$ restante presento alteración ecográfica en alguno de sus órganos, encontrándose una mayor frecuencia de afecciones en el tracto gastrointestinal, presentando alteración en 35 de las ecografías realizadas, lo que representa un $23.33 \%$, seguido por el hígado con un $16 \%$ y el bazo con el $8 \%$. Al evaluar la distribución de las patologías detectadas se encontró que la inflamación gástrica fue la patología más frecuente con un $8 \%$, seguida de hepatitis $(7,33 \%)$, cistitis $(5,33 \%)$ y gastroenteritis (4\%) (Tabla 2 ).

Tabla 2. Frecuencia de órganos afectados y sus principales lesiones diagnosticados mediante ecografía abdominal en la Clínica Veterinaria del Sur.

\begin{tabular}{|c|c|c|c|}
\hline Órgano & Patología & FA & $\mathbf{F R}$ \\
\hline \multicolumn{2}{|c|}{ Estomago e intestino } & 35 & $23,33 \%$ \\
\hline & Inflamación gástrica & 12 & $8 \%$ \\
\hline & Gastroenteritis & 6 & $4 \%$ \\
\hline & Cuerpo extraño & 5 & $3 \%$ \\
\hline & Enteritis & 5 & $3 \%$ \\
\hline & Colitis & 2 & $2 \%$ \\
\hline \multirow[t]{5}{*}{ Hígado } & & 24 & $16 \%$ \\
\hline & Hepatitis & 11 & $7,33 \%$ \\
\hline & Neoplasia hepática & 4 & $2,67 \%$ \\
\hline & Lipidosis hepática & 3 & $2 \%$ \\
\hline & Colangiohepatitis & 3 & $2 \%$ \\
\hline \multirow[t]{3}{*}{ Bazo } & & 12 & $8 \%$ \\
\hline & Esplenomegalia & 5 & $3,33 \%$ \\
\hline & Neoplasia esplénica & 4 & $2,67 \%$ \\
\hline \multirow[t]{8}{*}{ Riñones } & & 11 & $7,33 \%$ \\
\hline & Insuficiencia renal crónica & 4 & $2,67 \%$ \\
\hline & Insuficiencia renal aguda & 1 & $0.67 \%$ \\
\hline & Nefritis intersticial & 1 & $0.67 \%$ \\
\hline & Glomerulonefritis & 1 & $0.67 \%$ \\
\hline & Hidronefrosis & 1 & $0.67 \%$ \\
\hline & Fibrosis renal & 1 & $0.67 \%$ \\
\hline & Nefrolitiasis & 1 & $0.67 \%$ \\
\hline \multirow[t]{3}{*}{ Vejiga } & & 11 & $7,33 \%$ \\
\hline & Cistitis & 8 & $5,33 \%$ \\
\hline & Urolitiasis & 3 & $2 \%$ \\
\hline \multicolumn{2}{|c|}{ Otros* } & & $21.07 \%$ \\
\hline \multicolumn{2}{|c|}{ Sin hallazgos relevantes } & 26 & $17 \%$ \\
\hline
\end{tabular}

*corresponde a otros órganos afectados con una frecuencia de reporte de lesiones inferior al 5\%. FA=Frecuencia absoluta; FR= Frecuencia relativa 


\section{DISCUSIÓN}

Aunque muchos autores recalcan la importancia de la ecografía abdominal en la práctica médica para realizar un diagnóstico rápido y oportuno (INSTITUTO HISPANOAMERICANO DE GESTIÓN ACADÉMICA, 1999), no se encontraron estudios que reporten la frecuencia de patologías abdominales mediante ecografía.

En Colombia se han realizado estudios que reportan dentro de las principales alteraciones en la práctica clínica de pequeñas especies las alteraciones gastroentéricas (ALMANSA et al., 2007; HENAO et al., 2010;). Aunque el objetivo de este estudio no fue evaluar la casuística clínica, las alteraciones del tracto gastrointestinal también se encontraron dentro de las principales patologías diagnosticadas mediante ecografía, representando un $23,3 \%$ del total. Esto permite evidenciar la importancia del uso de esta herramienta diagnóstica en la práctica clínica, pues permitiría tener un análisis rápido, preciso y relativamente económico, aportando información acerca del grosor de las paredes intestinales, extensión de la lesión y motilidad del tracto digestivo, así como la visualización de importantes estructuras adyacentes como lo son linfonodulos y peritoneo (MOON LARSON y BILLER, 2009)

Algunos estudios han reportado que la ecografía de estómago, intestino delgado y colon es extremadamente útil en el diagnóstico de lesiones obstructivas, inflamaciones y enfermedades neoplásicas (MOON LARSON y BILLER, 2009), Se ha reportado una sensibilidad del $64 \%$ al usar la ecografía como medio diagnóstico de obstrucciones intestinales(PASTORE et al., 2007), aunque otros estudios reportan hasta un $85 \%$ de sensibilidad con una especificidad del $95 \%$ para esta misma patología (MANCZUR et al., 1998). En este estudio las principales patologías del tracto gastrointestinal diagnosticadas mediante ecografía correspondieron a alteraciones de tipo inflamatorio (gastritis, colitis, enteritis y gastroenteritis) y a cuerpos extraños. Según PENNINCK (2002), la inflamación gástrica es el hallazgo ecográfico más común en las enfermedades inflamatorias del tracto gastrointestinal, lo que se correlaciona con los hallazgos de este estudio donde la inflamación gástrica fue la alteración con mayor frecuencia de presentación, correspondiendo a un $8 \%$ del total de patologías diagnosticadas. Para realizar este diagnóstico se evalúa el espesor de la pared gástrica, que en un paciente canino normal debe ser de 3 a $5 \mathrm{~mm}$, mientras que en felinos se reporta de $2 \mathrm{~mm}$ entre los pliegues y 4,4 mm en región de los pliegues. Se considera que existe inflamación gástrica cuando el engrosamiento de la pared es mayor de $7 \mathrm{~mm}$ con preservación de las capas (PELLEGRINI, 2010).

Es importante informar que se ha demostrado que la ecografía no es una técnica útil para el diagnóstico de ulceraciones, perforaciones o ruptura del tracto gastrointestinal debido a que son fácilmente pasadas por alto y por tanto los resultados ecográficos deben ser interpretados con precaución en animales con signos clínicos compatibles con estos diagnósticos (PASTORE et al., 2007).

En este estudio el segundo sistema más frecuentemente afectado fue el hepatobiliar agrupando un $16 \%$ de las patologías diagnosticadas, seguido por el bazo con un $8 \%$. Algunos autores reportan una sensibilidad del $63 \%$ en la detección de lesiones hepáticas o esplénicas (PASTORE et al., 2007). Aunque el hígado es el órgano más grande de la cavidad abdominal su evaluación ecográfica es más difícil y subjetiva para los especialistas (PELLEGRINI, 2010), sin embargo, la ecografía como método rutinario para la exploración de las enfermedades hepáticas ha permitido ampliar el campo de las técnicas exploratorias del hígado brindando información referida a alteraciones estructurales que lo afectan, haciendo de la ecografía abdominal la modalidad de diagnóstico por imagen de primera elección en las patologías hepáticas (LOCKETT, 2009).

Finalmente, riñones y vejiga presentaron también una frecuencia importante de lesiones, contribuyendo cada uno con el $7,33 \%$ de las patologías diagnosticadas mediante ecografía abdominal, siendo la 
cistitis $(5,33)$ y la insuficiencia renal $(2,67 \%)$ las patologías principales que afectan estos órganos. Cuando la vejiga está llena, el espesor de su pared no debe sobrepasar los 2-3 mm, aunque la identificación de sus capas no siempre es posible. La cistitis se caracteriza por engrosamiento de la pared de la vejiga, local o difuso, no siempre fácil de evidenciar, a menos que la inflamación sea muy severa y crónica, casos en los cuales su espesor puede alcanzar los 6-8 mm. (G. González, s.f). La ecografía del tracto urogenital no sólo permite valorar el tracto urogenital sino también realizar toma de muestras eco guiadas (cistocentesis, citologías, biopsias) (SUÁREZ, 2017).
En conclusión la ecografía abdominal es una técnica versátil, económica y de gran utilidad en la medicina veterinaria de pequeñas especies, especialmente en alteraciones gastroentéricas, hepatobiliares, esplénicas y urinarias, permitiendo la orientación diagnóstica en la práctica clínica.

Este es el primer reporte sobre patologías diagnosticadas mediante ecografía abdominal realizado en Colombia, ya que sólo se trata de un registro de frecuencias es necesario estudios posteriores a nivel poblacional para conocer más a fondo los usos y potencialidades de esta técnica diagnóstica.

\section{REFERENCIAS}

ALMANSA M.,J.; GALÁN O,J.; BENAVI, O. 2007. Análisis retrospectivo de las historias clínicas de una. NOVA -Publicación Científica En Ciencias Biomédicas 5 (8):168-176.

DAYLE TYRRELL, C.B. 2006. Survey Of The Use Of Radiography Vs. Ultrasonography In The Investigation Of Gastrointestinal Foreign Bodies In Small Animals. Veterinary Radiology \& Ultrasound 47:404-408.

DIEZ BRU, N.; GARCIA, I.; PLEZE, P. 2004. Ecografía del tracto gastrointestinal en pequeños animales. Clínica veterinaria de pequeños animales, Vol. 24(2): 87-96. https://ddd. uab.cat/pub/clivetpeqani/11307064v24n2/11307064v24n2p87.pdf.

G. GONZÁLEZ, C. G. (s.f). Hallazgos Ecográficos Anormales de la Vejiga Urinaria en Caninos y Felinos. Facultad de Agronomía y Veterinaria, Universidad Nacional de Río Cuarto.

GARCÍA DE CASASOLA, G.; TORRES, J. 2012. Manual de Ecografía Clínica. Madrid: Sociedad Española de Medicina Interna. Madrid.

GARCIA, T.R. 2011. Ultrasonography of small intestinal obstructions: a contemporary approach. Journal of Small Animal Practice 52 (9):484-490.

HENAO VILLEGAS, S. ; TOJANCÍ DUQUE, C.P.; YÉPES CHAVARRIAGA, C.M.; USUGA SUÁREZ, A. 2010. Análisis retrospectivo de los registros clínicos del Centro de Veterinaria y Zootecnia de la Universidad CES 2004-2009. Revista CES Medicina Veterinaria y Zootecnia 5 (2):61-68.

INSTITUTO HISPANOAMERICANO DE GESTIÓN ACADÉMICA. 1999. I Curso Nacional Integral de Ecografía Ginecoobstétrica. Física de Ultrasonido y Ecografía de Abdomen. U. N.I. Académica. Recopilador. Lima, Peru.

LARSON, M.M. 2009. Ultrasound of the gastrointestinal tract. Ultrasound of the gastrointestinal tract.The Veterinary Clinics of the North America: Small Animal Practice. USA. 
LOCKETT, M.; KOSCINCZUK, P.; ROSCIANI, A.; INSFRÁN, R.; REPETTO, C. 2009. Diagnóstico ecográfico de afecciones hepáticas en caninos. Rev. vet. 20 (2):92-96.

MANCZUR, F.; VÖRÖS, K.; VRABÉLY, T.; WLADÁR, S.; NÉMETH, T.; FENYVES, B. 1998. Sonographic diagnosis of intestinal obstrucción in the dog. Acta Vet Hung. 46 (1):35-45

MAGLINTE, D.D. 2003. Radiology of small bowel obstruction: contemporary approach and controversies. Abdominal Imaging 3:160-178.

MANCZUR, F.; VÖRÖS, K.; VRABELY, T.; WLADÁR, S.; NÉMETH, T.; FENYVES, B. 1998. Sonographic diagnosis of intestinal obstrucción in the dog. Acta Vet. 46:35-45.

MARTÍNEZ, R. 1989. Ecografía clínica del abdomen. Jims:148-165.

MORENO BOISO, A.; HERVÁS RODRÍGUEZ, J.; CHACÓN, F. 2011. Significado patológico de la imagen ultrasónica en pequeños animales. Anales 24:214.

MOON LARSON, M.; BILLER, D.S. 2009 Ultrasound of the Gastrointestinal Tract. Vet Clin Small Anim. 39:747-759

NYLAND, T.G.; MATTOON, J.S.; HERRCESELL, E.; WISNER, E. 2001. Principios físicos. Págs. 1-86. En: Nyland, M.J. Saunders, T.G (Ed.). Small Animal Diagnostic Ultrasound. 2nd Edition. Saunder. Philadelphia.

PASTORE, G.E.; LAMB, C.R.; LIPSCOMB, V. 2007. Comparison of the results of abdominal ultrasonography and exploratory laparotomy in the dog and cat. J Am Anim Hosp Assoc 43:264-269.

PELLEGRINI, L.C.; SANTAROSA, I.M.; KROLIKOWSKI, G. 2010. Ultrassonografia do Abdome em Pequenos Animais. Ptags. 60-61. En: Pellegrini, I.S.L. (Ed.). Diagnóstico Por Imagem Em Medicina Veterinária. Universidade Federal de Santa Maria.

PENNINCK, D.G. 2002. Gastrointestinal tract. Págs. 207-.230. En: Nyland, M.J. Saunders, T.G (Ed.). Small Animal Diagnostic Ultrasound . 2nd edition. Saunders. Philadelphia.

SIMPSON JW, R.E. 1991. Digestive disease in the dog and cat. Blackwell Scientific Publications. USA.

SUÁREZ, M.; BERTOLANI, C.; AVELLANEDA, A.; TABAR, M. 2017. Las Vias Urinarias "Tan Sencillas Como Complejas". Disponible en: http://www.green-south.com/agro/ archivos/hallazgos ecograficos anormales vejiga urinaria caninos felinos.doc. Consultado: 20-09-2017.

XIFRA RUBIO, P.; GARCÍA SERRANO, S.I. 2015. Cómo preparar al paciente para una ecografía abdominal. Ateuves 17:24-33.

XIFRA RUBIO, P.; SERRANO GARCÍA, S.I. 2016. Ventajas de la ecografía sobre la radiología. Ateuves 17:24-33. 